\title{
Prognostic value of circulating tumor cells in the peripheral blood of patients with esophageal squamous cell carcinoma
}

This article was published in the following Dove Press journal:

OncoTargets and Therapy

3 March 2017

Number of times this article has been viewed

Yuanyuan Qiao, 1,* Jun Li, 2,* Chenghe Shi, ${ }^{1, *}$ Wei Wang, ${ }^{2}$ Xiuhua Qu,' Ming Xiong,' Yulin Sun, ${ }^{3}$ Dandan Li,' Xiaohang Zhao, ${ }^{1,3}$ Dajin Zhang'

'Center of Basic Medical Sciences, ${ }^{2}$ Department of Thoracic Surgery, Navy General Hospital of Chinese PLA, ${ }^{3}$ State Key Laboratory of Molecular Oncology, Cancer Institute and Hospital, Chinese Academy of Medical Sciences \& Peking Union Medical College, Beijing, People's Republic of China

*These authors contributed equally to this work Video abstract

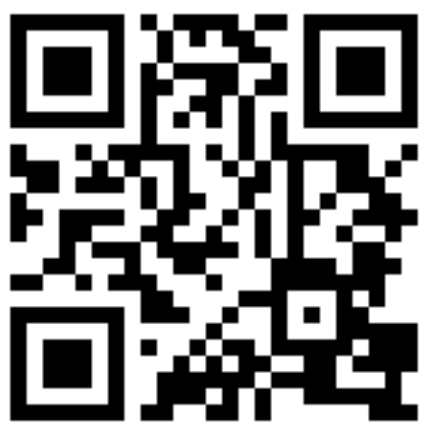

Point your SmartPhone at the code above. If you have a QR code reader the video abstract will appear. Or use: http://youtu.be/Y9vd-AIMTRA

Correspondence: Xiaohang Zhao

State Key Laboratory of Molecular Oncology, Cancer institute and Hospital, Chinese Academy of Medical Sciences \& Peking Union Medical College, 17 Panjiayuan Road, Chaoyang District, Beijing 10002I, People's Republic of China

Tel +86 I0 6695 I487

Email zhaoxhtao@।63.com

Dajin Zhang

Center of Basic Medical Sciences, Navy General Hospital of Chinese PLA, 6 Fucheng Road, Haidian District, Beijing 100048, People's Republic of Chin Tel +86 $106695 \quad 1485$

Email dajinzhang@sina.com
Objective: Circulating tumor cells (CTCs) of patients with malignant tumors can be used as a prognostic marker. However, there are few relevant reports to date on esophageal squamous cell carcinoma (ESCC). Our study assesses the clinical significance of CTCs in ESCC patients.

Patients and methods: CTCs were detected in 103 peripheral blood (PB) samples from 59 ESCC patients. Correlation between CTCs and clinical parameters was analyzed using the $\chi^{2}$ test or Fisher's exact test. Overall survival (OS) and progression-free survival (PFS) were analyzed using Kaplan-Meier analysis and univariate and multivariate methods.

Results: The CTC detection rate was 79.7\% (47/59) at baseline. The frequency of CTC-positive patients increased as the disease stage advanced (88.0\% in stages III-IV, 58.9\% in stages I-II). CTC counts $\geq 0 / 7.5 \mathrm{~mL}$ of $\mathrm{PB}$ were correlated with the degree of tumor differentiation, tumor infiltration, and lymph node and distant metastases. Overall, the OS and PFS of patients with CTC counts $\geq 3$ or $\geq 5 / 7.5 \mathrm{~mL}$ of $\mathrm{PB}$ before surgery were significantly shorter than those of patients with CTC counts $<3$ or $<5 / 7.5 \mathrm{~mL}$. Multivariate analysis showed CTC counts $\geq 5 / 7.5 \mathrm{~mL}$ of PB to be a strong prognostic indicator of OS (hazard ratio [HR] 12.478; 95\% confidence interval $[\mathrm{CI}], 8.2-34.3 ; P<0.05)$ and PFS (HR 6.524; 95\% CI, 1.2-34.3; $P<0.05$ ) in ESCC patients. Patients in whom CTCs changed from positive at baseline to a negative value after surgery had an excellent prognosis.

Conclusion: CTCs might serve as a reference indicator for the prognosis and monitoring of disease progression and treatment effects in ESCC.

Keywords: circulating tumor cells, esophageal squamous cell carcinoma, clinical significance, prognostic value

\section{Introduction}

The 4 most common cancers diagnosed in China are lung, stomach, liver, and esophageal cancers (ECs), accounting for $57 \%$ of all cases diagnosed in the country. ${ }^{1}$ Esophageal carcinoma is one of the most common and deadly cancers in China, with an incidence of $\sim 223,000$ new cases in 2012 (48.9\% of all worldwide cases), and esophageal squamous cell carcinoma (ESCC) is the main histological type. ${ }^{2-4}$ Due to the lack of early symptoms and reliable markers, the majority of patients are often diagnosed at an advanced stage. Furthermore, more than two-thirds of patients with ESCC suffer from relapse or distant metastasis, despite complete resection of the primary tumor and multimodal treatments. Indeed, patients with ESCC have an unfavorable prognosis, with the mean 5-year survival rate for all patients not exceeding $20 \% .^{5}$

Currently, the clinical diagnosis of ESCC is based on imaging screening, serological tumor markers, and histopathological methods. However, the sensitivity of 
imaging techniques for detection is limited for tumor lesions with a small diameter. Histopathology is the "gold standard" of tumor diagnosis, yet dynamical real-time monitoring cannot be realized due to the trauma to the body. In addition, although serum tumor markers, such as squamous cell carcinoma antigen (SCC-Ag), carcinoembryonic antigen (CEA), and fragments of cytokeratin-19 (CYFRA21-1), are commonly used for clinical diagnosis, the results are not very specific or reliable and are usually inconsistent with the disease status. Hence, effective auxiliary diagnosis methods are required to detect ESCC for monitoring therapeutic responses and for predicting outcomes.

Circulating tumor cells (CTCs), which are defined as cancer cells that have escaped from the primary tumor into the circulation, have great promise as a "liquid biopsy", ie, a noninvasive method of assessing tumor progression in real time. Indeed, the significance of CTCs in the peripheral blood (PB) of patients has been studied extensively in various malignancies. Many studies have demonstrated that CTCs are closely related to tumor metastasis, recurrence, and prognosis. ${ }^{6,7}$ In 2004, the United States Food and Drug Administration approved the CellSearch system for detecting CTCs in patients with advanced breast, prostate, and colorectal cancers, and CTC counts have been correlated with overall survival (OS) and progression-free survival (PFS) in these patients. ${ }^{8-11}$ Additionally, several studies have confirmed that the number of CTCs as well as their phenotypic changes in response to treatment can provide useful information for individual treatment.

To date, only a few studies have focused on CTCs in ESCC. ${ }^{12,13}$ Although nucleic acid-based methods have been utilized in these studies to detect representative markers of CTCs, such as CEA, CK19, and survivin, the morphology and number of CTCs cannot be detected due to the limitations of this approach. Detection of CTCs has recently been reported in the PB of ESCC patients. ${ }^{14}$ This study in Japan showed that the OS rate of patients with CTC counts $>0$ was significantly lower than that of patients without CTCs. The CTC number at the second-line assessment and therapeutic efficacy were highly related. However, the cutoff level was not clearly stated. Therefore, evidence for the prognostic role of CTCs in ESCC remains unclear, and the prognostic value of CTCs has not been defined. Accordingly, exploration of the significance of CTCs in ESCC is warranted, especially with regard to the high incidence of ESCC in China.

This study evaluated $103 \mathrm{~PB}$ samples from 59 patients with ESCC. The CTC diagnostic value and optimal CTC cutoff level for predicting OS and PFS in ESCC patients were investigated systematically. There were notable relationships between CTC counts and clinicopathological parameters when analyzing 3 different cutoff values (CTC counts 0,3 , and 5). Our data indicated that a high level of CTCs before surgery ( $\geq 3$ or 5 CTCs $/ 7.5 \mathrm{~mL}$ blood) was correlated with worse survival time (PFS and OS), and changes in CTCs before and after surgery were also correlated with patient PFS and OS. The results indicated that the presence of CTCs can serve as a novel indicator of a poor prognosis in ESCC patients. The number of CTCs was also evaluated as a prognostic parameter for patient clinical outcome and risk of relapse.

\section{Patients and methods}

\section{Study population and sample collection}

This study was performed at the Navy General Hospital of PLA (Beijing, People's Republic of China) and was approved by the ethics committee of Navy General Hospital of PLA. According to the rules of the ethics committee, all enrolled patients were required to sign and each enrolled patients have provided written informed consent for this study. Patients with histologically confirmed ESCC were eligible. A total of $7.5 \mathrm{~mL}$ of venous blood was drawn into BD Vacutainer tubes (BD, Franklin Lakes, NJ, USA) via central venipuncture; the first $2 \mathrm{~mL}$ of blood was discarded to avoid epithelial contamination from the venipuncture. Blood samples were collected before surgery and 7 days after surgery and processed at room temperature within $48 \mathrm{~h}$ (Figure 1).

We initiated blood sample collection in 2006. In total, 59 patients (mean age 61.5 years) with stages I-IV histologically proven ESCC were recruited for our study (51 males and 8 females). Tumor characteristics such as histological type, size, number of positive lymph nodes, and grade were obtained from pathology reports. From 2006 to 2011, 103 PB samples were collected from 59 patients with ESCC, 59 blood samples obtained before any therapy, and 44 blood samples drawn 7 days after surgery. The median follow-up duration was 5 years. Samples from 25 healthy volunteers comprised the negative control group. The healthy blood donors were aged between 25 years and 72 years and included 13 females and 12 males.

\section{CTC enrichment and slide preparation}

The collected blood samples were transferred to $50 \mathrm{~mL}$ centrifuge tubes (Corning Inc., Corning, NY, USA), and buffer 1 (137 mM NaCl, $2.7 \mathrm{mM} \mathrm{KCl,} 10$ mM Na 2 HPO4, 2 mM ethylenediaminetetraacetic acid (EDTA), 0.5\% BSA, pH 7.4) was added to a total volume of $50 \mathrm{~mL} \cdot{ }^{15-20}$ The tubes were centrifuged at $450 \times g$ for 5 minutes, and the supernatant was discarded. Erythrocyte lysis buffer $\left(155 \mathrm{mM} \mathrm{NH}_{4} \mathrm{Cl}, 10 \mathrm{mM}\right.$ 

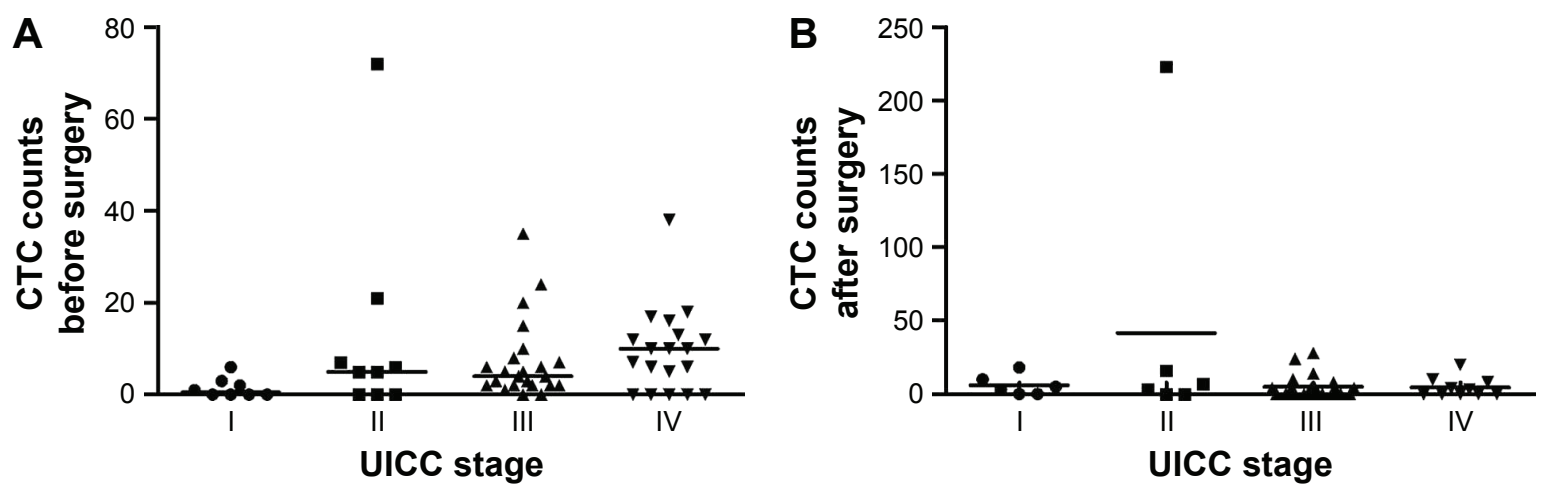

Figure I Distribution of the number of CTCs detected according to tumor stage in ESCC patients.

Notes: (A) Distribution of the number of CTCs detected in ESCC patients at different tumor stages before surgery. (B) Distribution of the number of CTCs detected in ESCC patients at different tumor stages after surgery.

Abbreviations: CTCs, circulating tumor cells; ESCC, esophageal squamous cell carcinoma; UICC, Union for International Cancer Control.

$\mathrm{KHCO}_{3}, 0.1 \mathrm{mM}$ EDTA) was added to a total volume of $50 \mathrm{~mL}$, and the cells were incubated at room temperature for 8 minutes in the dark under uniform velocity. The tubes were centrifuged at $450 \times g$, and the supernatant was discarded. The erythrocyte lysis process was repeated until the erythrocytes were sufficiently removed. The remaining cells were resuspended in $50 \mathrm{~mL}$ buffer 1 and counted. The tubes were centrifuged at $450 \times g$ for an additional 5 minutes, and the supernatant was discarded. Next, $20 \mu \mathrm{L}$ of antihuman CD45-coated magnetic beads (Miltenyi Biotec, Bergisch Gladbach, Germany) was added per $10^{7}$ cells, and the cell suspension was mixed and maintained at $4^{\circ} \mathrm{C}$ for 15 minutes. Next, $10 \mathrm{~mL}$ of buffer 1 was added prior to centrifugation at $450 \times g$, and the supernatant was discarded. To resuspend the cells, $500 \mu \mathrm{L}$ of buffer 1 was added per $10^{7}$ cells, and the cell suspension was loaded onto an LS separation column (Miltenyi Biotec). The effluent was collected through a strong magnetic field (theoretically containing tumor cell components) and loaded evenly onto a PAP pen-outlined area (30×30 mm) (Sigma-Aldrich Corp., St Louis, MO, USA) on a glass slide (Thermo Fisher Scientific, Waltham, MA, USA). The slides were kept at room temperature to air dry. The cells were then fixed in $2 \%$ paraformaldehyde for 40 minutes and rinsed 3 times with phosphate-buffered saline (PBS).

\section{Immunofluorescence staining}

The slides were rinsed twice with PBS. The cells were permeabilized with $0.1 \%$ TritonX-100-PBS and blocked with $2 \%$ BSA-PBS at room temperature for 30 minutes. Double immunofluorescence staining was performed with $100 \mu \mathrm{L}$ of CK8/18/19-anti-cytokeratin-fluorescein isothiocyanate (anti-cytokeratin-FITC) (1:100, green) and anti-CD45phycoerythrin $(1: 1,000$, red; Miltenyi Biotec) for indirect immunofluorescence staining. The cells were blotted dry and mounted with medium containing the nuclear dye 4',6-diamidino-2-phenylindole (DAPI; Sigma-Aldrich
Corp.). ${ }^{15,16,18} \mathrm{~A}$ blind review of the 3-color fluorescent images by 3 technicians confirmed CTC identity.

\section{Identification of CTCs}

The diameter of the cells was larger than $10 \mu \mathrm{m}$, and the outline of the cells was completely visible by green fluorescence microscopy. ${ }^{18,21,22}$ The criteria for CTCs based on fluorescent images included CK8/18/19 positivity, DAPI positivity, and absence of CD45 expression.

\section{Statistical analysis}

Associations between the detection of CTCs and the baseline clinical characteristics and OS or PFS of the patients were analyzed. This statistical analysis was conducted using the SPSS 16.0 software package. The $\chi^{2}$ test or Fisher's exact test was used to compare differences in CTC detection rates among patients in the different groups according to different factors (ie, tumor stage, size, lymph node metastasis, and distant metastasis). Kaplan-Meier survival curves were then used to describe the survival distributions of patients with different levels of CTCs. Moreover, the patients were followed up for $\sim 5$ years. All analyses were performed for 3 different cutoff values: CTCs $\geq 0, \geq 3$, and $\geq 5$. PFS was defined as the time from blood collection to the time of progression or death. OS was defined as the time from blood collection to death. The log-rank test was used to compare survival rates between each group. Cox proportional hazards regression was used to obtain univariate and multivariate hazard ratios for PFS and OS. A 2-sided test was used, and a $P$-value of $\leq 0.05$ indicated a statistically significant difference.

\section{Results}

Incidence of CTCs in patients with ESCC

At baseline (before surgery), 59 blood samples were obtained, and CTC counts $>0$ per $7.5 \mathrm{~mL}$ of blood were detected in 
47 patients $(79.7 \%)$. Of these, 4 of 8 patients were at stage I $(50.0 \%), 6$ of 9 were at stage II $(66.7 \%), 21$ of 23 were at stage III (91.3\%), and 14 of 19 were at stage IV (73.7\%). CTC counts $\geq 3$ were detected in 37 patients $(62.7 \%)$, and $\geq 5$ CTCs were detected in 32 patients (54.2\%; Figure 1). For every $7.5 \mathrm{~mL}$ of $\mathrm{PB}$, the average number of CTCs was 3 in patients with well-differentiated tumors; in contrast, the average number of CTCs was 13 in patients with poorly differentiated tumors. In addition, the average number of CTCs detected in patients with no lymph node invasion was 4 , whereas the average in those with lymph node invasion was 10. The average number of CTCs detected in patients with no distant metastasis was 5 , but it was 15 in patients with distant metastasis.

CTC analysis was performed on postoperative day 7 for 44 patients. CTCs were detected in 26 of these patients (59.1\%), as follows: 4 of 5 in stage I $(80.0 \%), 4$ of 6 in stage II (66.7\%), 13 of 23 in stage III (56.5\%), and 5 of 10 in stage IV (50.0\%). The CTC count was $\geq 3$ in 24 patients $(54.5 \%)$ and $\geq 5$ in 14 patients $(31.8 \%$; Figure 1$)$.

CTC analysis was performed in 25 healthy volunteers as a negative control group, and no CTCs were found in the blood specimens of these healthy volunteers.

\section{Association between CTC count and clinicopathological variables}

The patients with ESCC were categorized according to certain clinical parameters, and the association of various clinical characteristics with the presence of CTCs before surgery was analyzed for 3 different cutoff values (CTC counts $\geq 0, \geq 3$, and $\geq 5$ ). In these correlation analyses, no significant association was observed between CTC count and gender, age, tumor size, and location of the primary tumor. However, the analysis did confirm the positive association between a higher number of CTCs and lymph node metastasis $(P<0.05$ for CTC thresholds $\geq 0,3$, and $5 \mathrm{CTCs} / 7.5 \mathrm{~mL}$ blood). Pathologic stage was also associated with the number of CTCs $(P<0.05$ for CTC thresholds $\geq 3$ and $\geq 5 \mathrm{CTCs} / 7.5 \mathrm{~mL}$ blood). Moreover, an increased number of CTCs was observed in patients with tumors of a certain depth, ie, T2-4 compared to T0-1. Tumor differentiation was also dramatically associated with CTC count when differentiation was divided into high and low levels ( $P<0.05$ for CTC thresholds $\geq 0,3$, and $5 \mathrm{CTCs} / 7.5 \mathrm{~mL}$ of blood). CTC count was also associated with distant metastasis $(P<0.05$ for CTC thresholds $\geq 3$ and $5 \mathrm{CTCs} / 7.5 \mathrm{~mL}$ of blood; Table 1).

\section{Association between CTC count and survival time (PFS and OS) of ESCC patients}

Fifty-seven patients with ESCC were followed up in this study (another 2 patients were lost during follow-up). The mean follow-up time was 5 years, and a total of 30 deaths occurred during this period. The mortality rates were $65.2 \%(30 / 46)$, $78.4 \%$ (29/37), and $87.5 \%$ (28/32), respectively, for patients with $>0,>3$, and $>5$ CTCs per $7.5 \mathrm{~mL}$ of blood before surgery. Preoperative detection of CTC levels (CTC count $\geq 3$ and 5 CTCs $/ 7.5 \mathrm{~mL}$ blood) was associated with both OS and PFS (Figure 2 and Table 2). OS and PFS were significantly shorter for patients with $\geq 3$ CTCs compared with those with $<3$ (mean time; 447 days vs 889 days for OS, 419 days vs 859 days for PFS, $P=0.002$ ) or $\geq 5$ (mean time; 385 days vs 911 days for OS, 348 days vs 875 days for PFS, $P<0.001$; Table 3). Conversely, the number of CTCs present after surgery was not a strong predictor of PFS and OS.

\section{CTC count as a predictor of survival time (PFS and OS) in ESCC patients}

Univariate Cox regression analyses were initially used to evaluate known prognosticators of the risk of death in the study cohort. The results showed that gender, age, tumor location, tumor differentiation, and tumor size were not associated with significantly shorter OS and PFS. Conversely, the CTC level prior to surgery ( $\geq 3$ or $5 \mathrm{CTCs} / 7.5 \mathrm{~mL}$ blood) as well as lymph node metastasis, distant metastasis, and pathologic stage were significantly associated with both PFS and OS.

Multivariate Cox regression analysis was performed to examine the clinical factors that were significant in the univariate analysis. The preoperative detection of a CTC count $\geq 5$ per $7.5 \mathrm{~mL}$ blood remained a strong predictor of OS (hazard ratio [HR] 12.5; 95\% confidence interval [CI] 8.2-34.3; $P<0.05$ ) and PFS (HR 6.5; 95\% CI 1.2-34.3; $P<0.05)$. The risk of mortality in the presurgical group with $\geq 5$ CTCs was 12.478 times (OS) and 6.524 times (PFS) higher than in the group with $<5$ CTCs. Therefore, the strong association of preoperative CTCs and OS as well as PFS was confirmed for a cutoff of $\geq 5$ CTCs per $7.5 \mathrm{~mL}$ of blood (Table 4). In addition, the results showed lymph node metastasis, metastasis, and pathologic stage (all $P$-values $<0.05$ ) to be associated with significantly shortened OS and PFS. These were significant prognostic factors in this study. 
Table I Correlation between CTC count (per $7.5 \mathrm{~mL}$ peripheral blood) preoperation and clinicopathological date of ESCC

\begin{tabular}{|c|c|c|c|c|c|c|c|}
\hline Prognostic factors & Total & CTCs $>0$ (\%) & CTCs $=0$ (\%) & CTCs $\geq 3$ (\%) & CTCs <3 (\%) & CTCs $\geq 5$ (\%) & CTCs $<5$ (\%) \\
\hline \multicolumn{8}{|l|}{ Gender } \\
\hline Male & 51 & $41(80.4)$ & $10(19.6)$ & $33(64.7)$ & $18(35.3)$ & $27(52.9)$ & $24(47.1)$ \\
\hline Female & 8 & $6(75.0)$ & $2(25.0)$ & $6(75.0)$ & $2(25.0)$ & $6(75.0)$ & $2(25.0)$ \\
\hline$P$-value & & 0.725 & & 0.567 & & 0.243 & \\
\hline \multicolumn{8}{|l|}{ Age (years) } \\
\hline$\geq 65$ & 18 & $17(94.4)$ & I (5.6) & $14(77.8)$ & $4(22.2)$ & $14(77.8)$ & $4(22.2)$ \\
\hline$<65$ & 41 & $30(73.2)$ & II (26.8) & $25(61.0)$ & $16(39.0)$ & $25(61.0)$ & $16(39.0)$ \\
\hline$P$-value & & 0.062 & & 0.209 & & 0.759 & \\
\hline \multicolumn{8}{|l|}{ Pathologic tumor size } \\
\hline$\geq 3 \mathrm{~cm}$ & 45 & 37 (82.2) & $8(17.8)$ & $34(75.6)$ & II (24.4) & $31(68.9)$ & $14(3 \mid .1)$ \\
\hline$<3 \mathrm{~cm}$ & 14 & $10(7 \mid .4)$ & $4(28.6)$ & $9(64.3)$ & $5(35.7)$ & $8(57.1)$ & $6(42.9)$ \\
\hline$P$-value & & 0.381 & & 0.407 & & 0.417 & \\
\hline \multicolumn{8}{|l|}{ Tumor location } \\
\hline Upper & 4 & $4(100.0)$ & $0(0.0)$ & $3(75.0)$ & I (25.0) & $2(50.0)$ & $2(50.0)$ \\
\hline Middle & 28 & $20(7 \mathrm{I} .4)$ & $8(28.6)$ & $17(60.7)$ & II (39.3) & I $4(50.0)$ & $14(50.0)$ \\
\hline Lower & 27 & $23(85.2)$ & $4(14.8)$ & $19(70.4)$ & $8(29.6)$ & $17(63.0)$ & $10(37.0)$ \\
\hline$P$-value & & 0.259 & & 0.696 & & 0.607 & \\
\hline \multicolumn{8}{|l|}{ Tumor differentiation } \\
\hline High differentiation & 38 & 27 (7I.I) & II (28.9) & $16(42.1)$ & $22(57.9)$ & $19(50.0)$ & $19(50.0)$ \\
\hline Low differentiation & 21 & $20(95.2)$ & I (4.8) & $20(95.2)$ & I (4.8) & $18(85.7)$ & $3(14.3)$ \\
\hline$P$-value & & $0.027^{\#}$ & & $0^{\#}$ & & $0.007^{\#}$ & \\
\hline \multicolumn{8}{|l|}{ Tumor depth } \\
\hline $\mathrm{TO}+\mathrm{TI}$ & 8 & $4(50.0)$ & $4(50.0)$ & $2(25.0)$ & $6(75.0)$ & I (I2.5) & $7(87.5)$ \\
\hline $\mathrm{T} 2+\mathrm{T} 3+\mathrm{T} 4$ & 51 & $43(84.3)$ & $8(I 5.7)$ & $36(70.6)$ & $15(29.4)$ & $31(60.8)$ & $20(39.2)$ \\
\hline$P$-value & & $0.025^{\#}$ & & $0.012^{\#}$ & & $0.011^{\#}$ & \\
\hline \multicolumn{8}{|l|}{ Lymph node status } \\
\hline Negative & 35 & $24(68.6)$ & II (3I.4) & $19(54.3)$ & $16(45.1)$ & $17(48.6)$ & $18(5 \mid .4)$ \\
\hline Positive & 24 & $23(95.8)$ & I (4.2) & $20(83.3)$ & $4(16.7)$ & 19 (79.2) & $5(20.8)$ \\
\hline$P$-value & & $0.01 I^{\#}$ & & $0.02 I^{\#}$ & & $0.009^{\#}$ & \\
\hline \multicolumn{8}{|l|}{ Tumor metastasis } \\
\hline $\begin{array}{l}\text { Other organs } \\
\text { metastasis }\end{array}$ & 11 & $10(90.9)$ & I (9.1) & $10(90.9)$ & $\mathrm{I}(9.1)$ & $10(90.9)$ & I (9.1) \\
\hline No metastasis & 48 & $37(77.1)$ & II (22.9) & $28(58.3)$ & $20(4 \mid .7)$ & $22(45.3)$ & $26(54.2)$ \\
\hline$P$-value & & 0.304 & & $0.042^{\#}$ & & $0.007^{\#}$ & \\
\hline \multicolumn{8}{|l|}{ Pathologic stage } \\
\hline$I+I I$ & 17 & $10(58.9)$ & $7(4 \mid .2)$ & $6(34.5)$ & II (65.5) & $4(25.0)$ & $13(75.0)$ \\
\hline $\mathrm{III}+\mathrm{IV}$ & 42 & $35(83.3)$ & $7(16.7)$ & 31 (73.3) & II (26.7) & $29(69.0)$ & $13(33.3)$ \\
\hline$P$-value & & $0.027^{\#}$ & & $0.014^{\#}$ & & $0.003^{\#}$ & \\
\hline
\end{tabular}

Notes: No CTC was detected in 25 healthy volunteers. "Significant relationship $(P<0.05)$ was found between progression status and this group using the 2 -sided $\chi^{2}$ test. Abbreviations: CTC, circulating tumor cell; ESCC, esophageal squamous cell carcinoma.

\section{Association between CTC changes and survival time (PFS and OS) in ESCC patients}

Blood specimens were obtained from 44 patients after surgery, and univariate analysis showed a CTC count $\geq 3$ to be associated with a shorter OS. Patients with CTC changes pre- and postsurgery exhibited differences in OS and PFS. Among all patients, 17 were positive for CTCs both pre- and postsurgery, 7 changed from CTC positive to negative, and 9 were negative for CTCs both pre- and postsurgery. Analysis of OS and PFS based on changes in CTC counts from baseline to postsurgery demonstrated that patients who were negative for CTCs or changed from CTC positive to negative lived longer than those who were consistently positive for CTCs. The average OS of patients who remained CTC positive after surgery, who changed from CTC positive to negative, and with 0 CTC before treatment was 375 days, 713 days, and 787 days, respectively; the average PFS of patients who were CTC positive, who changed from CTC positive to negative and with 0 CTC before treatment, was 285 days, 720 days, and 654 days, respectively. Patients who were CTC positive before and after treatment exhibited significantly poorer OS 

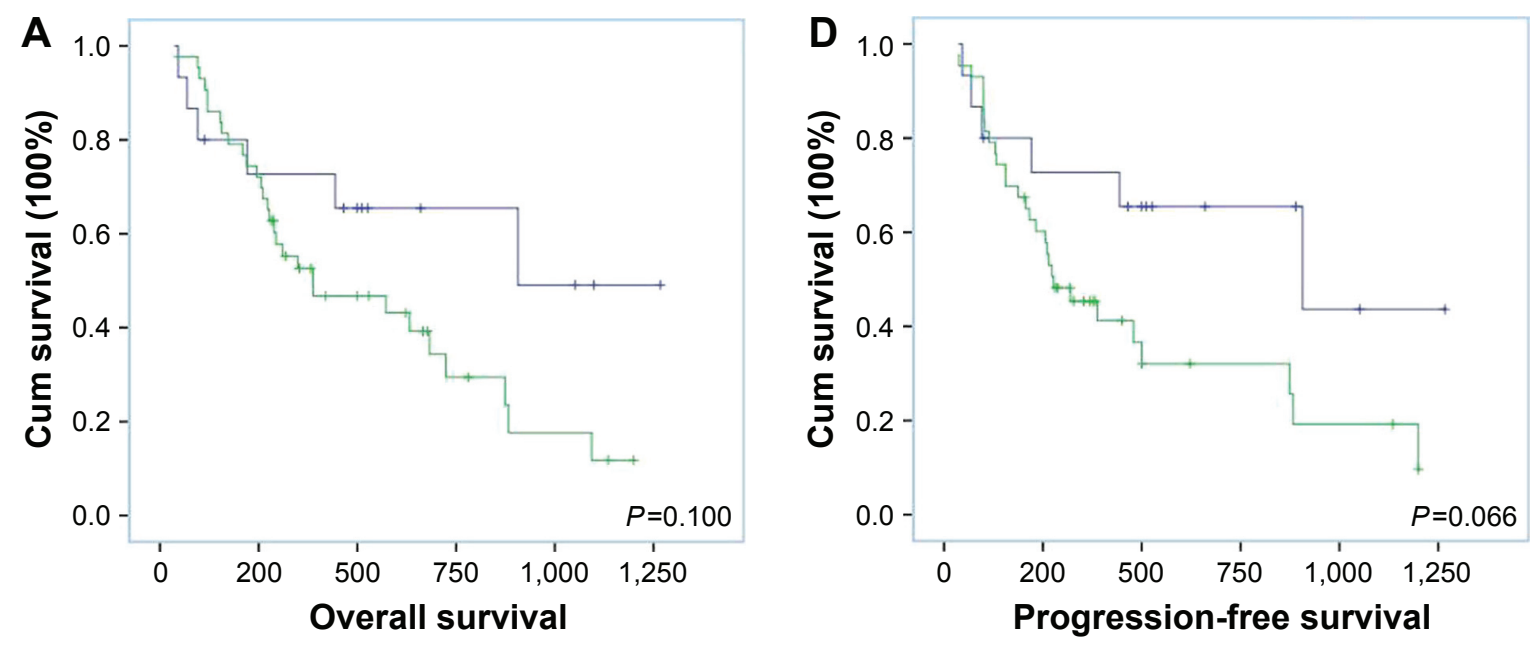

$\neg \mathrm{CTC}=0 \backsim \mathrm{CTC}>0+\mathrm{CTC}=0$-censored $+\mathrm{CTC}>0$-censored
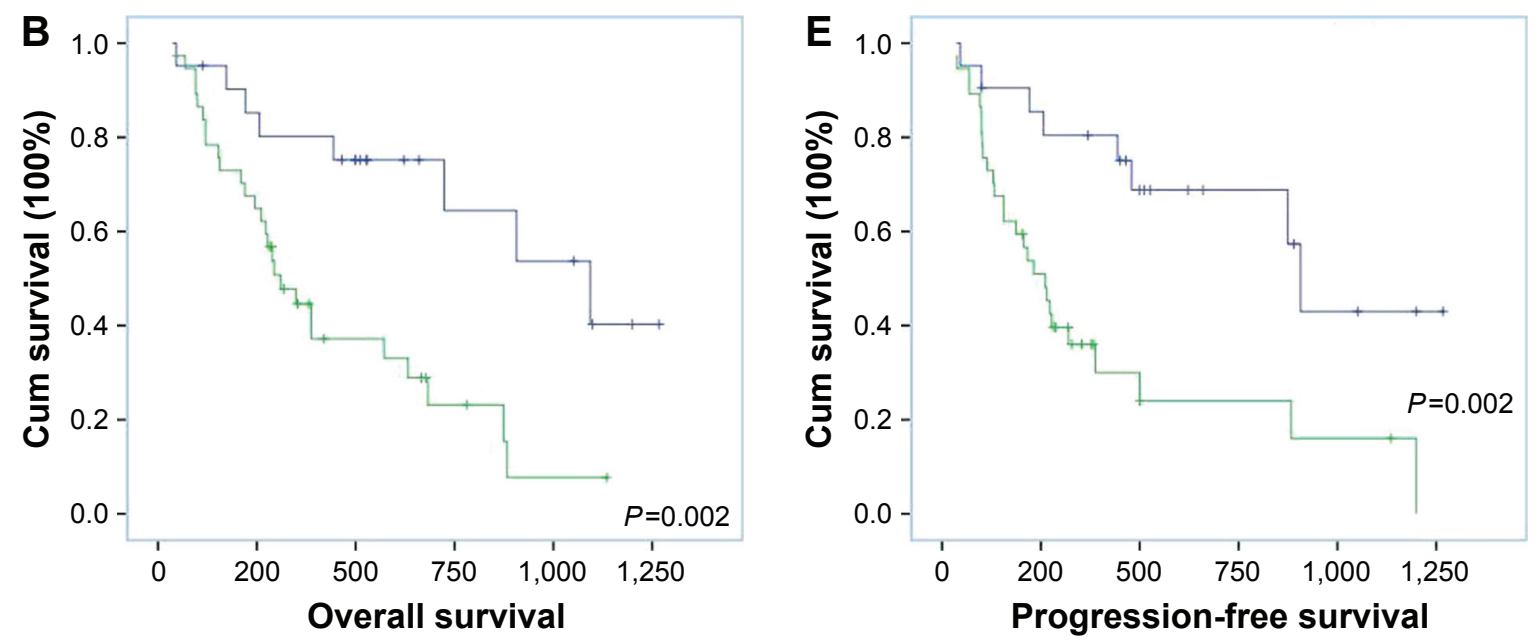

$\neg \mathrm{CTC}<3-\neg \mathrm{CTC} \geq 3+\mathrm{CTC}<3$-censored + CTC $\geq 3$-censored
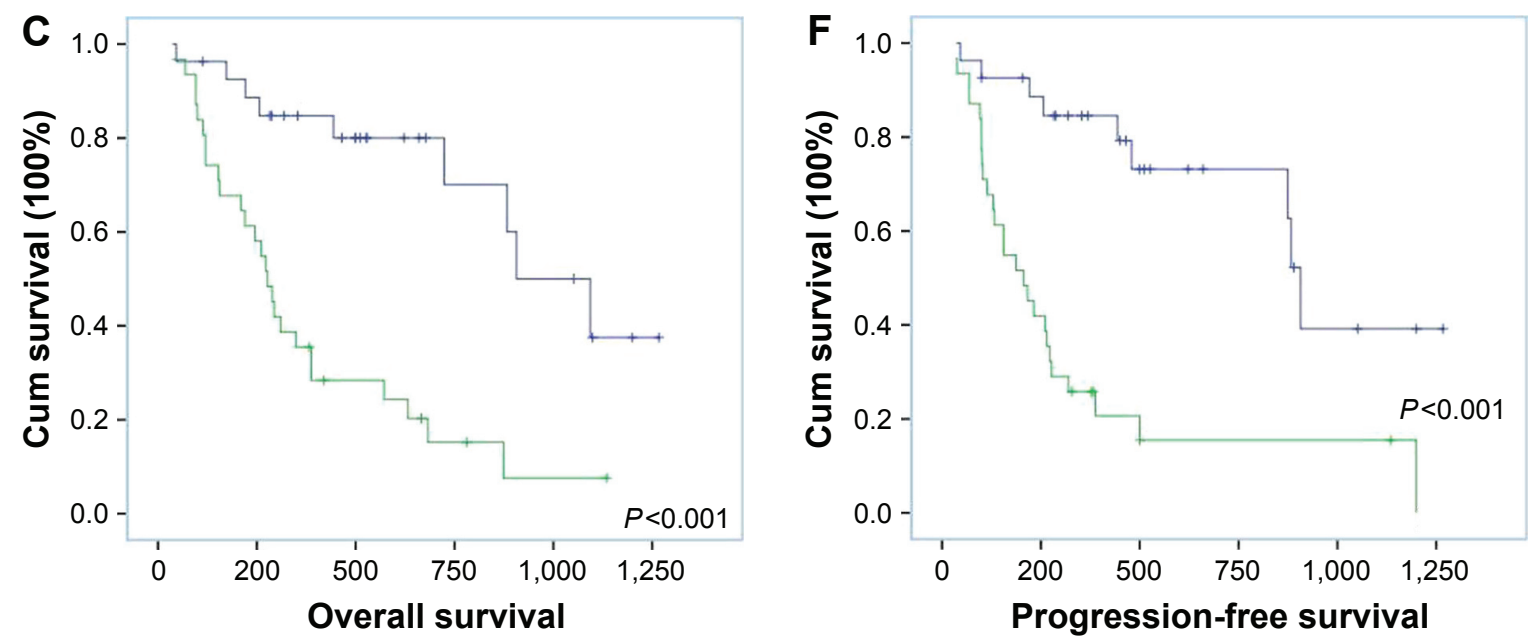

$$
\neg \text { CTC }<5 \neg \text { CTC } \geq 5+\text { CTC }<5 \text {-censored }+ \text { CTC } \geq 5 \text {-censored }
$$

Figure 2 OS and PFS according to different CTC cutoffs.

Notes: Kaplan-Meier estimates of OS (A-C) and PFS (D-F) in patients with ESCC. OS and PFS were significantly shorter in patients with CTC counts $\geq 3$ or 5 compared with those with CTC counts $<3$ or $5(P<0.05)$.

Abbreviations: OS, overall survival; PFS, progression-free survival; CTC, circulating tumor cell; ESCC, esophageal squamous cell carcinoma; Cum, cumulative. 
Table 2 Survival time according to the status of CTCs before operation $(n=58)$

\begin{tabular}{|c|c|c|c|c|c|c|c|}
\hline CTC groups & Total & Overall survival & $\chi^{2}$ & $P$-value & Progression-free survival & $\chi^{2}$ & $P$-value \\
\hline $\mathrm{CTC}=0$ & 15 & $832.830 \pm|36.39|$ & 2.7 & 0.1 & $813.194 \pm 138.525$ & 3.387 & 0.066 \\
\hline $\mathrm{CTC}>0$ & 43 & $543.199 \pm 63.657$ & & & $496.572 \pm 73.912$ & & \\
\hline СТC $<3$ & 21 & $889.305 \pm|33.23|$ & 9.607 & $0.002^{\wedge}$ & $859.802 \pm 107.207$ & 9.899 & $0.002^{\wedge}$ \\
\hline CTC $\geq 3$ & 37 & $447.658 \pm 59.774$ & & & $419.616 \pm 78.597$ & & \\
\hline СТC $<5$ & 27 & $911.149 \pm 89.084$ & 17.055 & $<0.001^{\wedge}$ & $875.978 \pm 94.570$ & $17.66 \mid$ & $<0.001^{\wedge}$ \\
\hline $\mathrm{CTC} \geq 5$ & 31 & $385.935 \pm 58.98 \mid$ & & & $348.929 \pm 74.806$ & & \\
\hline
\end{tabular}

Notes: Data are presented as mean \pm SD. ^Significant relationship $(P<0.05)$ was found between different groups using Kaplan-Meier analysis.

Abbreviations: CTC, circulating tumor cell; SD, standard deviation.

and PFS than patients whose CTC counts changed from positive to negative (Figure 3 and Table 5).

\section{Discussion}

Recently, CTCs isolated from blood have been evaluated as a new prognostic tool and as a marker for patients of various types of solid tumors. ${ }^{23,24}$ Many studies have shown that the number and characterization of CTCs may provide valuable information of clinical relevance when used in conjunction with imaging studies. ${ }^{25}$

The TNM staging system is the foundation of cancer treatment and prognosis. However, as TNM staging does not include cytology and molecular biology factors, we cannot accurately understand the existence of the tumor state of the host using only this measure, which emphasizes the need for better diagnostic and staging biomarkers to inform therapy methods. CTCs, which possess characteristics of cancer cells, may be utilized for tumor staging classification and for the screening of high-risk tumors. For example, it has been demonstrated that CTC counts differ for various stages and subtypes of breast cancer, and detection of CTCs in PB was more frequently observed in advanced than in early stages. ${ }^{26,27}$ In addition, the percentage of non-small-cell lung cancer (NSCLC) patients with an absolute majority of CTCs in the blood significantly increased from stages I-II to IV..$^{28}$ Another study revealed that evaluation of CTCs is a promising indicator for predicting tumor prognosis and the clinical efficacy of chemotherapy in patients with ESCC. ${ }^{14}$
Our study proves the clinical significance of CTCs as a preoperative staging parameter in ESCC. In this study, 103 PB samples from 59 ESCC patients were investigated. The median follow-up time was $\sim 5$ years; 59 PB samples were collected before primary tumor resection and 44 samples at day 7 after surgery. The CTC average detection rate in the 59 presurgical patients was $79.6 \%$ (47/59), with $50.0 \%$ (4/8) for early-stage and $84.3 \%$ (43/51) for advanced-stage patients $(P=0.025)$. Overall, more CTCs were detected with more extensive tumor burden, and the probability of being positive for the presence of CTCs was significantly associated with the cancer stage.

We assessed the relationship between CTC characteristics and clinicopathological factors or disease prognosis. Previous studies have demonstrated that poor cell differentiation and a high TNM stage are associated with a high positive rate of CTCs. Matsushita et al ${ }^{14}$ studied 90 patients with ESCC and reported that the presence of CTCs was significantly correlated with distant metastases, such as pleural dissemination and hematogenous metastasis. In addition, Rahbari et $\mathrm{al}^{29}$ performed a meta-analysis on the influence of disseminated tumor cells in bone marrow on the prognosis of patients with colorectal cancer. Our results suggested a strong association between CTCs and the stage of ESCC (poor differentiation, lymph node status, tumor differentiation, depth, and metastasis). The observed sensitivity of CTCs at least $\geq 3$ suggests that these cells may be a highly sensitive biomarker for detecting disease stage, and detection of more CTCs

Table 3 Survival time according to the status of CTCs after operation $(n=44)$

\begin{tabular}{|c|c|c|c|c|c|c|c|}
\hline CTC groups & Total & Overall survival & $\chi^{2}$ & $P$-value & Progression-free survival & $\chi^{2}$ & $P$-value \\
\hline $\mathrm{CTC}=0$ & 18 & $682.787 \pm 98.374$ & 3.898 & 0.06 & $680.58 \mathrm{I} \pm \mathrm{I} I 2.470$ & 3.875 & 0.052 \\
\hline $\mathrm{CTC}>0$ & 26 & $388.570 \pm 55.084$ & & & $289.717 \pm 42.594$ & & \\
\hline СТC $<3$ & 20 & $688.997 \pm 101.039$ & 4.127 & 0.04 & $683.311 \pm 117.717$ & 3.121 & 0.077 \\
\hline $\mathrm{CTC} \geq 3$ & 22 & $387.383 \pm 50.194$ & & & $311.453 \pm 42.624$ & & \\
\hline СТC $<5$ & 30 & $637.477 \pm 83.632$ & 3.691 & 0.06 & $621.397 \pm 94.698$ & 3.867 & 0.064 \\
\hline $\mathrm{CTC} \geq 5$ & 14 & $339.143 \pm 49.263$ & & & $271.103 \pm 54.788$ & & \\
\hline
\end{tabular}

Notes: Data are presented as mean \pm SD. There were no significant correlations between different groups using Kaplan-Meier analysis. Abbreviations: CTC, circulating tumor cell; SD, standard deviation. 


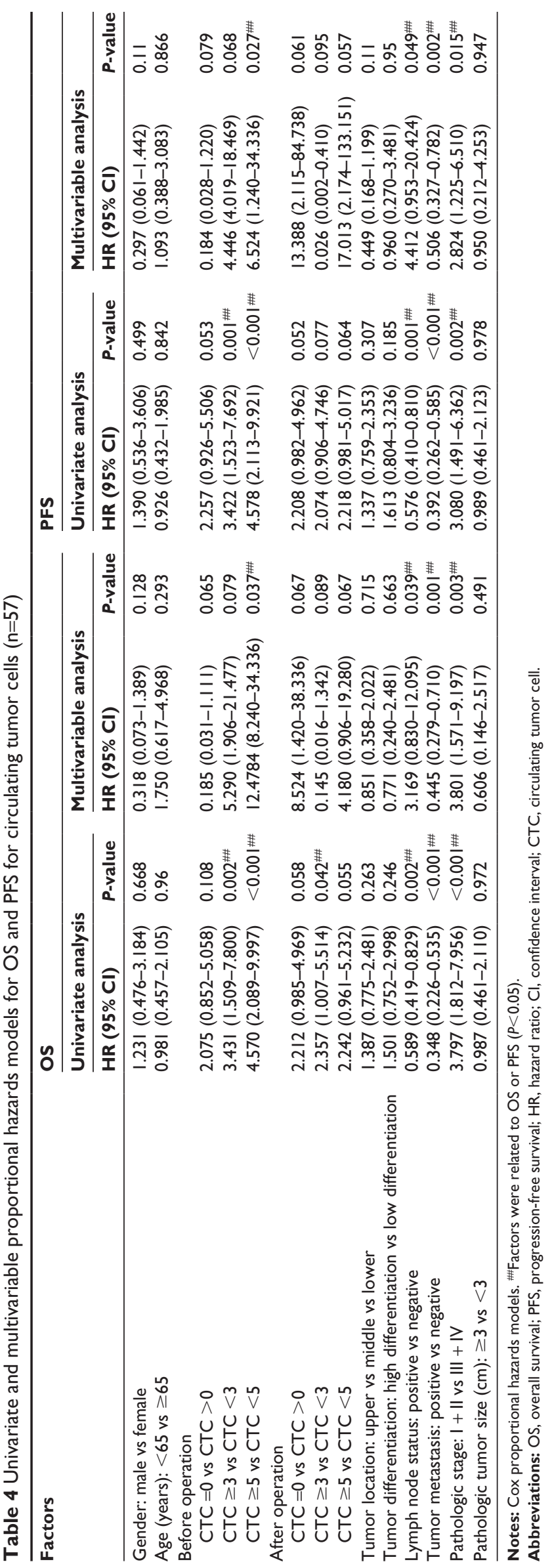

was associated with low-grade differentiation and lymph node status. Our results also showed that the positivity rate of CTCs did not differ significantly with regard to gender, age, tumor size, and location. Based on our study, a CTC threshold of at least $3 \mathrm{CTCs} / 7.5 \mathrm{~mL}$ can be applied to evaluate the relationship between $\mathrm{CTC}$ number and clinical factors of ESCC.

CTCs are necessary for tumor recurrence. These cells have undergone the epithelial-mesenchymal transition (EMT), a key process involved in metastasis and recurrence. ${ }^{30}$ During EMT, cells exhibit reduced adhesion, obtain a mesenchymal phenotype, and enter into the peripheral circulation. When arriving at an appropriate organ, CTCs escape the vascular system, cross the basement membrane, and proliferate at the new site, forming a metastasis. Currently, up to two-thirds of patients develop local or distant tumor metastases after resection, and $>90 \%$ die of tumor recurrence and distant metastasis. ${ }^{13}$ CTCs appear to be responsible for distant metastasis and relapse. According to our study, CTC detection in PB can be clinically informative for tumor recurrence and metastasis, for monitoring treatment response in patients with advanced cancer as well as in patients with early localized cancer. ${ }^{31}$

Our results showed an association between CTC count and the presence of metastasis, and the results for patients with ESCC demonstrated that the presence of $\geq 5$ CTCs can predict tumor recurrence and decreased survival time. The mean follow-up time was 5 years, and the CTC counts were in accordance with imaging studies at several time points. Further validation of CTC enumeration could facilitate the monitoring of disease status and recurrence and provide support for treatment decisions. ${ }^{32}$ Our group has also reported the malignant characteristics of CTCs and the corresponding molecular features of the primary tumor in a patient with ESCC. Before surgery, 7 intact CTCs and 12 CTCs with a fragmented membrane were detected, and the patient died after 6 months as a result of lung metastasis. These results suggest that the malignant characteristics of CTCs during the disease process may predict tumor burden and the risk of relapse and metastasis. ${ }^{15}$

CTC enumeration is valuable in many prospective studies as a prognostic marker for some cancer types, such as breast, prostate, colorectal, lung, and ovarian cancers. ${ }^{33,34}$ Reeh et al reported that CTC-positive patients with metastatic EC had a significantly shorter OS than CTC-negative patients. ${ }^{13}$ Another study reported that metastatic breast cancer patients with $\geq 5$ CTCs in $7.5 \mathrm{~mL}$ of blood at baseline had a poor OS time. ${ }^{35}$ In colorectal cancer, a value of $\geq 3$ CTCs has commonly been used as a cutoff, and Cohen et $\mathrm{al}^{11}$ reported that the median PFS and OS of patients with metastatic 

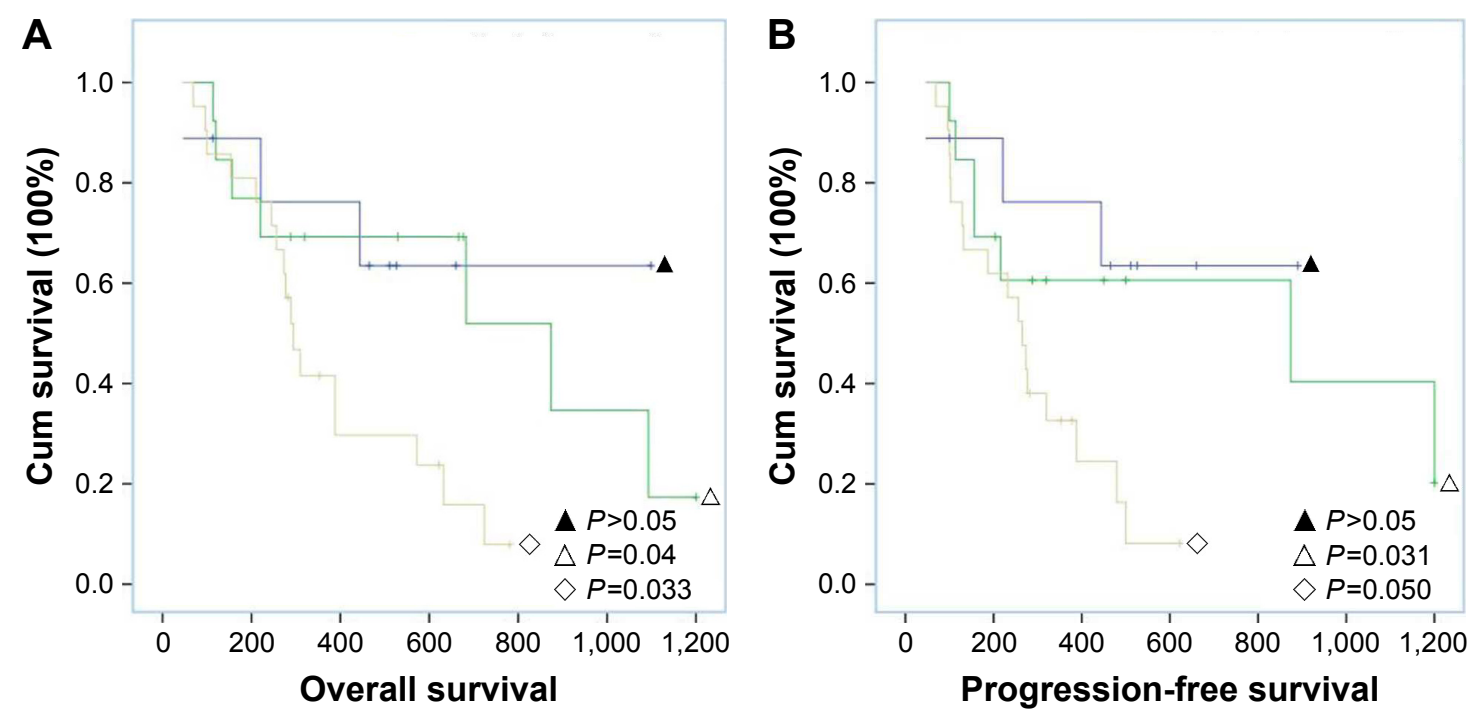

$\begin{array}{lll}\neg \text { Negative before operation } & \neg \text { Positive } \rightarrow \text { negative } & \rightarrow \text { Positive } \rightarrow \text { positive } \\ + \text { Negative before operation-censored } & + \text { Positive } \rightarrow \text { negative-censored } & \rightarrow \text { Positive } \rightarrow \text { positive-censored }\end{array}$

Figure 3 Blood samples were collected from 44 patients both pre- and postsurgery.

Notes: According to OS (A) and PFS (B), patients with CTCs who remained positive for CTCs pre- and postsurgery exhibited a poorer prognosis than those who were positive for CTCs and then negative for CTC postsurgery. A significant difference in PFS was observed between patients who were negative for CTCs before surgery and those who remained positive at both pre- and postsurgery for CTCs.

Abbreviations: OS, overall survival; PFS, progression-free survival; CTC, circulating tumor cell; Cum, cumulative.

colorectal cancer were nearly twice as high for patients with a few CTCs $(<3 \mathrm{CTCs} / 7.5 \mathrm{~mL}$ blood) relative to patients with elevated CTCs ( $\geq 3$ CTCs). In addition, Bitting et al ${ }^{36}$ reported that a high CTC count (CTCs $\geq 5$ ) can be used to define an unfavorable prognosis in prostate cancer.

Compared with other types of tumors, the detection and evaluation of CTCs in ESCC are rare. To our knowledge, this study is the first prospective evaluation of CTC thresholds for predicting survival time (OS and PFS) in patients with ESCC. OS and PFS were similarly influenced by the presence of CTCs and were significantly shorter with higher numbers of CTCs. Based on univariate Cox regression analyses, $\geq 5$ CTCs predicted a poorer prognosis than 0 CTC. We found that the optimum threshold for prediction (OS and PFS) using CTCs was $\geq 5$ before surgery. Lymph node status, tumor metastasis, pathologic stage, and CTC level before

Table 5 Association between CTC changes and survival time in ESCC patients

\begin{tabular}{lllll}
\hline $\begin{array}{l}\text { CTC } \\
\text { groups }\end{array}$ & OS & P-value & PFS & $P$-value \\
\hline I vs 2 & 787.333 vs 713.269 & 0.684 & 654.635 vs 720.250 & 0.629 \\
I vs 3 & 787.333 vs 375.534 & 0.067 & 654.635 vs 285.497 & $0.023^{\wedge \wedge}$ \\
2 vs 3 & 713.269 vs 375.534 & $0.033^{\wedge \wedge}$ & 720.250 vs 285.497 & $0.05^{\wedge \wedge}$ \\
\hline
\end{tabular}

Notes: I: negative before operation $\rightarrow$ negative after surgery; 2: positive before operation $\rightarrow$ negative after surgery; 3 : positive before operation $\rightarrow$ positive after surgery. ${ }^{\wedge}$ There are significant differences in OS or PFS between the groups.

Abbreviations: CTC, circulating tumor cell; ESCC, esophageal squamous cell carcinoma; OS, Overall survival; PFS, progression-free survival. surgery ( $\geq 3$ or $5 / 7.5 \mathrm{~mL}$ blood) were significantly associated with both PFS and OS. Multivariate Cox regression was utilized to investigate factors associated with survival time, and the results showed that $\mathrm{CTC} \geq 5 / 7.5 \mathrm{~mL}$ blood, lymph node status, presence of metastasis, and pathologic stage were associated with significantly shortened OS and PFS. ESCC patients with CTC $\geq 5 / 7.5 \mathrm{~mL}$ blood had a 12.5 -fold increased risk of a shorter OS compared to patients with CTC $<5$ and, similarly, had a 6.5 -fold increased risk of a shorter PFS compared to patients with CTC $<5$. Additional analysis revealed lymph node status, presence of metastasis, and pathologic stage to be associated with shortened OS and PFS. We conclude that the level of CTCs is a strong predictor of survival time, and an optimal cutoff of $\geq 5$ CTCs per $7.5 \mathrm{~mL}$ of blood was confirmed to predict OS or PFS.

Transition from an unfavorable baseline CTC level to a favorable CTC level after surgery has a strong impact on survival. Patients with such conversion showed a favorable OS in small-cell lung cancer. ${ }^{37}$ Based on multivariate analysis, we also found that a change in CTC detection from positive to negative may be a stronger indicator of OS and PFS; indeed, the survival time of patients whose CTC number changed from positive to negative was close to that of patients without CTCs at baseline (OS: 787.333 vs 713.269 , $P=0.684$; PFS: 654.635 vs $720.250, P=0.629)$. Furthermore, patients with a CTC number that stayed positive before and after treatment showed significantly poorer PFS than patients 
whose CTC changed from positive to negative $(P=0.033)$. In contrast, postoperative number of CTCs and survival time had no obvious correlation in our study (Figure 3 ).

Such results involving CTC detection using an immunomagnetic system have not been reported before, especially for ESCC. Nonetheless, there were several limitations in our study. The major limitation was the small sample size, and we could thus not effectively apply statistical methods to every subgroup of patients, leading to low statistical power for some variables in the stratified subgroups. In addition, the threshold value was derived from a cohort at a single institution and was not validated in multicenter trials.

\section{Acknowledgments}

We warmly thank Ms Fengjiao Liu for providing the clinical information. This work was supported by grants from the Nature Science Foundation of China (project no 31500756 and 81472350 ).

\section{Disclosure}

The authors report no conflicts of interest in this work.

\section{References}

1. Chen W, Zheng R, Baade PD, et al. Cancer statistics in China, 2015. CA Cancer J Clin. 2016;66(2):115-132.

2. Li C, Li DC, Che SS, et al. The decreased expression of miR-625 predicts poor prognosis of esophageal squamous cell carcinoma. Int $J$ Clin Exp Med. 2015;8(6):9560-9564.

3. Zhao P, Dai M, Chen W, Li N. Cancer trends in China. Jpn J Clin Oncol. 2010;40(4):281-285.

4. IAFROC. Oesophageal Cancer Estimated Incidence, Mortality and Prevalence Worldwide in 2012. Available from GLOBOCAN 2012, Available from: http://globocan.iarc.fr/Default.aspx. Accessed February 22, 2017.

5. Song Y, Li L, Ou Y, et al. Identification of genomic alterations in oesophageal squamous cell cancer. Nature. 2014;509(7498):91-95.

6. Wang N, Shi L, Li H, et al. Detection of circulating tumor cells and tumor stem cells in patients with breast cancer by using flow cytometry: a valuable tool for diagnosis and prognosis evaluation. Tumour Biol. 2012; 33(2):561-569.

7. Hou JM, Krebs M, Ward T, et al. Circulating tumor cells as a window on metastasis biology in lung cancer. Am J Pathol. 2011;178(3):989-996.

8. Lucci A, Hall CS, Lodhi AK, et al. Circulating tumour cells in nonmetastatic breast cancer: a prospective study. Lancet Oncol. 2012;13(7): 688-695.

9. de Bono JS, Scher HI, Montgomery RB, et al. Circulating tumor cells predict survival benefit from treatment in metastatic castration-resistant prostate cancer. Clin Cancer Res. 2008;14(19):6302-6309.

10. Rink M, Chun FK, Minner S, et al. Detection of circulating tumour cells in peripheral blood of patients with advanced non-metastatic bladder cancer. BJU Int. 2011;107(10):1668-1675.

11. Cohen SJ, Punt CJ, Iannotti N, et al. Relationship of circulating tumor cells to tumor response, progression-free survival, and overall survival in patients with metastatic colorectal cancer. J Clin Oncol. 2008;26(19): 3213-3221.

12. Yin XD, Yuan X, Xue JJ, Wang R, Zhang ZR, Tong JD. Clinical significance of carcinoembryonic antigen-, cytokeratin 19-, or survivinpositive circulating tumor cells in the peripheral blood of esophageal squamous cell carcinoma patients treated with radiotherapy. Dis Esophagus. 2012;25(8):750-756.
13. Reeh M, Effenberger KE, Koenig AM, et al. Circulating tumor cells as a biomarker for preoperative prognostic staging in patients with esophageal cancer. Ann Surg. 2015;261(6):1124-1130.

14. Matsushita D, Uenosono Y, Arigami T, et al. Clinical significance of circulating tumor cells in peripheral blood of patients with esophageal squamous cell carcinoma. Ann Surg Oncol. 2015;22(11):3674-3680.

15. Ren C, He P, Zhang J, Zheng Z, Qiao Y, Zhao X. Malignant characteristics of circulating tumor cells and corresponding primary tumor in a patient with esophageal squamous cell carcinoma before and after surgery. Cancer Biol Ther. 2011;11(7):633-638.

16. Grover PK, Cummins AG, Price TJ, Roberts-Thomson IC, Hardingham JE. Circulating tumour cells: the evolving concept and the inadequacy of their enrichment by EpCAM-based methodology for basic and clinical cancer research. Ann Oncol. 2014;25(8):1506-1516.

17. Balasubramanian P, Lang JC, Jatana KR, et al. Multiparameter analysis, including EMT markers, on negatively enriched blood samples from patients with squamous cell carcinoma of the head and neck. PLoS One. 2012;7(7):e42048.

18. Konigsberg R, Obermayr E, Bises G, et al. Detection of EpCAM positive and negative circulating tumor cells in metastatic breast cancer patients. Acta Oncol. 2011;50(5):700-710.

19. $\mathrm{Mu} \mathrm{H}$, Lin KX, Zhao H, et al. Identification of biomarkers for hepatocellular carcinoma by semiquantitative immunocytochemistry. World J Gastroenterol. 2014;20(19):5826-5838.

20. Guo W, Yang XR, Sun YF, et al. Clinical significance of EpCAM mRNA-positive circulating tumor cells in hepatocellular carcinoma by an optimized negative enrichment and qRT-PCR-based platform. Clin Cancer Res. 2014;20(18):4794-4805.

21. Lapin M, Tjensvoll K, Oltedal S, et al. MINDEC-an enhanced negative depletion strategy for circulating tumour cell enrichment. Sci Rep. 2016;6:28929

22. Gao Y, Zhu Y, Zhang Z, Zhang C, Huang X, Yuan Z. Clinical significance of pancreatic circulating tumor cells using combined negative enrichment and immunostaining-fluorescence in situ hybridization. $J$ Exp Clin Cancer Res. 2016;35:66.

23. Giuliano M, Giordano A, Jackson S, et al. Circulating tumor cells as prognostic and predictive markers in metastatic breast cancer patients receiving first-line systemic treatment. Breast Cancer Res. 2011;13(3):R67.

24. Hartkopf AD, Wagner P, Wallwiener D, Fehm T, Rothmund R. Changing levels of circulating tumor cells in monitoring chemotherapy response in patients with metastatic breast cancer. Anticancer Res. 2011;31(3): 979-984.

25. Chinen LT, de Carvalho FM, Rocha BM, et al. Cytokeratin-based CTC counting unrelated to clinical follow up. J Thorac Dis. 2013;5(5): 593-599.

26. Bidard FC, Peeters DJ, Fehm T, et al. Clinical validity of circulating tumour cells in patients with metastatic breast cancer: a pooled analysis of individual patient data. Lancet Oncol. 2014;15(4):406-414.

27. Li L, Liu Y, Zhang S, et al. [Detection of circulating tumor cells and its clinical value for different stages and various subtypes of breast cancer]. Zhonghua Yi Xue Za Zhi. 2014;94(36):2812-2815.

28. Yin J, Wang Y, Yin H, et al. Circulating tumor cells enriched by the depletion of leukocytes with bi-antibodies in non-small cell lung cancer: potential clinical application. PLoS One. 2015;10(8):e0137076.

29. Rahbari NN, Reissfelder C, Muhlbayer M, et al. Correlation of circulating angiogenic factors with circulating tumor cells and disease recurrence in patients undergoing curative resection for colorectal liver metastases. Ann Surg Oncol. 2011;18(8):2182-2191.

30. Bourcy M, Suarez-Carmona M, Lambert J, et al. Tissue factor induced by epithelial-mesenchymal transition triggers a procoagulant state that drives metastasis of circulating tumor cells. Cancer Res. 2016; 76(14):4270-4282.

31. Stott SL, Lee RJ, Nagrath S, et al. Isolation and characterization of circulating tumor cells from patients with localized and metastatic prostate cancer. Sci Transl Med. 2010;2(25):25ra23.

32. Qiao YY, Lin KX, Zhang Z, et al. Monitoring disease progression and treatment efficacy with circulating tumor cells in esophageal squamous cell carcinoma: a case report. World J Gastroenterol. 2015;21(25):7921-7928. 
33. Poveda A, Kaye SB, McCormack R, et al. Circulating tumor cells predict progression free survival and overall survival in patients with relapsed/recurrent advanced ovarian cancer. Gynecol Oncol. 2011; 122(3):567-572.

34. Ramirez JM, Fehm T, Orsini M, et al. Prognostic relevance of viable circulating tumor cells detected by EPISPOT in metastatic breast cancer patients. Clin Chem. 2014;60(1):214-221.

35. Cristofanilli M, Budd GT, Ellis MJ, et al. Circulating tumor cells, disease progression, and survival in metastatic breast cancer. $N$ Engl J Med. 2004;351(8):781-791.
36. Bitting RL, Healy P, Halabi S, George DJ, Goodin M, Armstrong AJ. Clinical phenotypes associated with circulating tumor cell enumeration in metastatic castration-resistant prostate cancer. Urol Oncol. 2015;33(3):110.e1-110.e9.

37. Naito T, Tanaka F, Ono A, et al. Prognostic impact of circulating tumor cells in patients with small cell lung cancer. J Thorac Oncol. 2012;7(3): $512-519$.

\section{Publish your work in this journal}

OncoTargets and Therapy is an international, peer-reviewed, open access journal focusing on the pathological basis of all cancers, potential targets for therapy and treatment protocols employed to improve the management of cancer patients. The journal also focuses on the impact of management programs and new therapeutic agents and protocols on

\section{Dovepress}

patient perspectives such as quality of life, adherence and satisfaction. The manuscript management system is completely online and includes a very quick and fair peer-review system, which is all easy to use. Visit http://www.dovepress.com/testimonials.php to read real quotes from published authors.

Submit your manuscript here: http://www.dovepress.com/oncotargets-and-therapy-journal 